\title{
Balance retraining outcomes in a 74-year-old adventitiously deafened adult with bilateral vestibulopathy: a case report
}

\begin{abstract}
Inner ear (audio-vestibular mechanism) dysfunction may result in comorbidities of; hearing impairment, dizziness, vertigo, and imbalance. Many times, these symptoms are not mutually exclusive, and a myriad of symptoms arise from acute pathologies or trauma to the inner ear. This system is vulnerable across an individual's lifespan from infant to older adult. In this case study, we will examine a case of a 74-year-old male with bilateral vestibular dysfunction and subsequent history of falls and imbalance. His childhood history is of interest, as he was deafened by spinal meningitis in infancy, post-language acquisition. In July 2020, he presented to our clinic at the American Institute of Balance (AIB), in Largo, Florida, USA, for complaints of progressively worsened balance function since 2017. As is the AIB protocol, a multidisciplinary approach included comprehensive neurodiagnostic and physical therapy assessment. Neurodiagnostic test results indicated a bilateral vestibulopathy. He was subsequently treated by the vestibular-trained Physical Therapy team for four weeks with clinically significant improvements in his Dizziness Handicap Inventory and objective scores for postural stability. Despite the absence of fully intact vestibular function, post-treatment outcomes revealed significant improvement on standardized subjective and objective measurements. This supports the efficacy of providing balance retraining therapy to individuals with demonstrable and irreversible bilateral vestibular deficiencies as a valid means to reduce their falls risk and improve their quality of life for activities of daily living.
\end{abstract}

Keywords: imbalance, bilateral vestibulopathy, disequilibrium, American sign language, deaf, balance retraining therapy, rehabilitation, substitution.
Volume 5 Issue 5 - 2020

\author{
Steven E Spinks,' Brittany M Fauble,' Richard \\ E Gans ${ }^{1,2}$ \\ 'Vice President of Research, The American Institute of Balance, \\ USA \\ ${ }^{2}$ Nova Southeastern University and University of South Florida, \\ USA
}

Correspondence: Steven E Spinks, PT, DPT, OCS, COMT, FAAOMPT,Vice President of Research, The American Institute of Balance, USA, Tel 727.398.5728, Fax 72739849I4, Emails.spinks@dizzy.com

Received: September 02, 2020 | Published: September 23 2020
Abbreviations: DHI, dizziness handicap inventory; CDP, computerized dynamic posturography; VOR, vestibular-ocular reflex; SOP, sensory organization performance;

\section{Introduction}

Dizziness and imbalance account for $4 \%$ of all emergency room visits and $20 \%$ of neurology consultation..$^{1-3}$ It is the third most common symptom for emergency room consultation following headache and cerebrovascular accidents. ${ }^{4} \mathrm{Up}$ to 800,000 individuals are hospitalized due to injuries related to falls in the United States alone. ${ }^{5}$ It is known that this places a burden on the healthcare system and costs the United States, on average, $\$ 50$ billion dollars annually. ${ }^{6}$ The balance system consists of three main components required to maintain the fight against the inevitable, gravity: proprioception, visual system, and the vestibular system. Each one of these systems places an intricate role in communicating to the central nervous system, the brain, to prepare the body for an appropriate motor output to maintain our upright and safe posture. While each component of this system is able to function in isolation, it is best utilized with proper communication from the others so the brain can make the right decision for balance.

In the event of one or more of these systems not functioning properly, or at full capacity, the other components will work to adapt via compensatory shift or substitution. This adaptation requires complex inputs from the sensory systems and rewiring from the brain for this proper output. The gold standard treatment for many vestibulopathies is the utilization of Diagnosis-Based Strategies. In the case for vestibular rehabilitation, often it is discussed that adaptation and habituation principles act on the brain for compensatory mechanisms. When both vestibular apparatuses are dysfunctional, central compensation is even more challenging. This is when the patient will undergo a principle called substitution. This is a welldocumented rehabilitation concept for the utilization of remaining systems and strengthening them through balance and physical therapy techniques. ${ }^{8,9}$

\section{Case description}

\section{History}

A 74-year-old male presents with a primary concern of progressive imbalance. He was previously active prior to his onset of symptoms in 2017; including deep-sea fishing for days at a time and regular, moderate-level exercise. Symptoms of imbalance are described as progressive with an onset most noticeably around April 2017. Two resulting falls, without significant head or bodily injury reported. His daily activities have been affected including his ability to ambulate on dynamic surfaces and in the dark. His imbalance has resulted in him becoming sedentary with significant deconditioning. While he denies any preceding event, illness, or change in medication to his onset of symptoms in 2017 , he does have a history of chemo- and radiationtherapy for throat cancer in 2008; he denies any immediate changes to his balance function following the aforementioned cancer treatments. 
Bilateral vestibulopathy and disequilibrium is reported in the literature secondary to chemotherapy. ${ }^{10}$ Medical history is remarkable for hypertension and acquired Deafness due to spinal meningitis at 2.5 years of age, post language acquisition. Lightheadedness, dizziness, vertigo, and orthopedic concerns were all denied during the history and intake portion of the evaluation. His primary language is American Sign Language (ASL) and all assessment, management, and rehabilitation were conducted in ASL.

\section{Examination}

The patient was seen at The American Institute of Balance, a multidisciplinary clinic in Largo, Florida, USA. A thorough, evidencebased neurodiagnostic examination by our vestibular audiologist was completed prior to physiotherapy. A detailed examination was performed; subjective examination included subjective questionnaires, past medical history, and current complaints. Of note, the subjective questionnaires included the Dizziness Handicap Inventory. The Dizziness Handicap Inventory is a 25 -item, well validated, selfreported questionnaire that is predictive of functional balance impairment ${ }^{12}$ secondary to vestibular impairment. The patient's baseline DHI yielded a moderate subjective impairment at 52\%. Post-treatment, the DHI was repeated and yielded a $4 \%$ subjective impairment which exceeds both the minimal detectable change (MDC) and the minimally clinical important difference (MCID) for this test. ${ }^{13}$ Table 1 summarizes all examination findings from this patient. The patient underwent two separate evaluations during his plan of care. His initial evaluation was performed by a vestibular audiologist that performed the neurodiagnostic examination detailed later in his publication. The second evaluation was performed by a vestibular physiotherapist.

Table I Summary of examination findings: initial evaluation and discharge

\begin{tabular}{|c|c|c|c|c|c|}
\hline & & \multicolumn{2}{|c|}{$\begin{array}{l}\text { Initial } \\
\text { evaluation }\end{array}$} & \multicolumn{2}{|c|}{ Discharge } \\
\hline Subjective & $\mathrm{DHI}$ & \multicolumn{2}{|l|}{$52 \%$} & \multicolumn{2}{|l|}{$4 \%$} \\
\hline \multirow[t]{3}{*}{$\begin{array}{l}\text { Objective } \\
\text { Physiotherapy }\end{array}$} & TUG & \multicolumn{2}{|c|}{$10.28 \mathrm{~s}$} & \multicolumn{2}{|l|}{$8.38 \mathrm{~s}$} \\
\hline & 30STS & \multicolumn{2}{|l|}{9} & \multicolumn{2}{|l|}{16} \\
\hline & MMT & \multicolumn{2}{|c|}{ 04-May } & \multicolumn{2}{|l|}{$5-/ 5$} \\
\hline \multirow{11}{*}{$\begin{array}{l}\text { Neuro } \\
\text { diagnostic }\end{array}$} & Gans SOP & \multicolumn{2}{|l|}{+} & \multicolumn{2}{|l|}{ - } \\
\hline & & Left & Right & Left & Right \\
\hline & CDP & + & + & - & - \\
\hline & $\begin{array}{l}\text { Kinetic } \\
\text { Rotary Chair }\end{array}$ & + & + & + & + \\
\hline & Oculomotors & - & - & - & - \\
\hline & Gaze & - & - & - & - \\
\hline & Headshake & - & - & - & - \\
\hline & Side-Lying & - & - & - & - \\
\hline & Hallpike & - & - & - & - \\
\hline & Positional & - & - & - & - \\
\hline & $\begin{array}{l}\text { Bithermal } \\
\text { Calorics }\end{array}$ & + & + & + & + \\
\hline
\end{tabular}

Abbreviations: DHI, dizziness handicap inventory; CDP, computerized dynamic posturography; SOP, sensory organization performance;TUG, Timed Up and Go Test; 30STS, 30 Second Sit to Stand Test; MMT, Manual Muscle Testing

\section{Neurodiagnostic examination}

Postural stability was evaluated with the sensory organization test (SOT) of computerized dynamic posturography (CDP) and the Gans Sensory Organization Performance Test (Gans SOP). The Gans SOP is a bedside evaluation tool that quantifies the participation of the three systems of equilibrium; proprioception, vision, and the vestibular system. It is a combination of the Romberg, tandem Romberg, clinical test of sensory integration of balance (CTSIB), and Fukuda Stepping Tests; it can also be utilized as an outcome measure. 5 The pattern of sway deviation and falls in both CDP and Gans SOP tests were suggestive of multifactorial involvement. This pattern is frequently seen in patients with bilateral vestibular involvement and subsequent deconditioning, and is often improved with balance retraining therapy, including strengthening and conditioning exercises, with the addition of substitution Vestibular Rehabilitation Therapy (VRT) protocols. ${ }^{14}$ Following one month of rehabilitation, the CDP and Gans SOP tests were repeated and found to be within age and height normalized parameters. See Figure 1 for results and explanation of findings for the Gans SOP pre- and post-rehabilitation. See Figure 2 for results and explanation of findings for the initial evaluation and post therapy CDP results. Of note, the DHI has significant correlations with the sensory organization sub-test of platform posturography ${ }^{13}$ and our pre and post-treatment measurements were in agreement
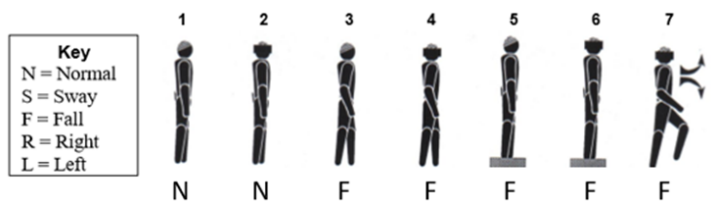

Figure IA Fall on conditions 3-6 and Stepping Fukuda (condition 7) of the Gans SOP at the initial assessment and prior to treatment shows a multifactorial pattern of disequilibrium.

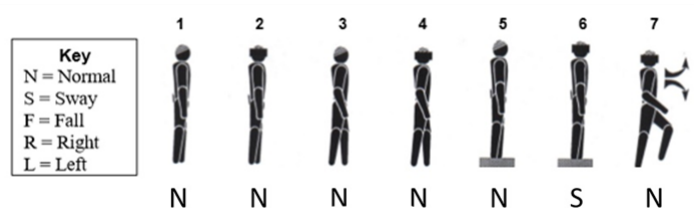

Figure IB Post-treatment Gans SOP findings are within normal limits when compared to age and height matched peers.

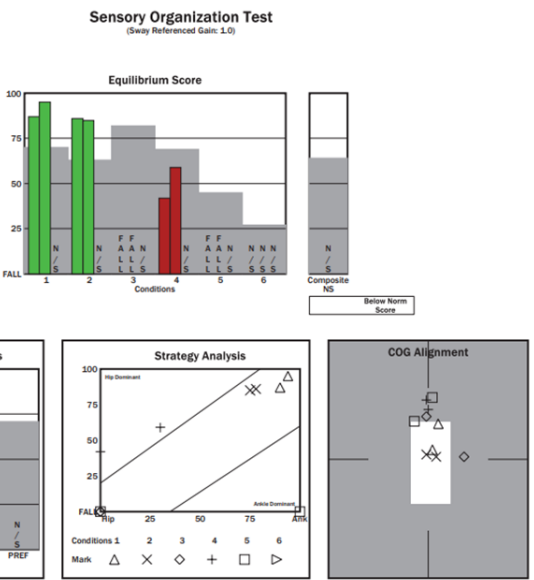

Figure 2A Baseline (pre-treatment) sensory organization test (SOT) of computerized dynamic posturography (CDP) testing shows a multifactorial pattern of disequilibrium. Of note, condition 6 was not completed due to the patient's fatigue and falls on conditions 3-5. 

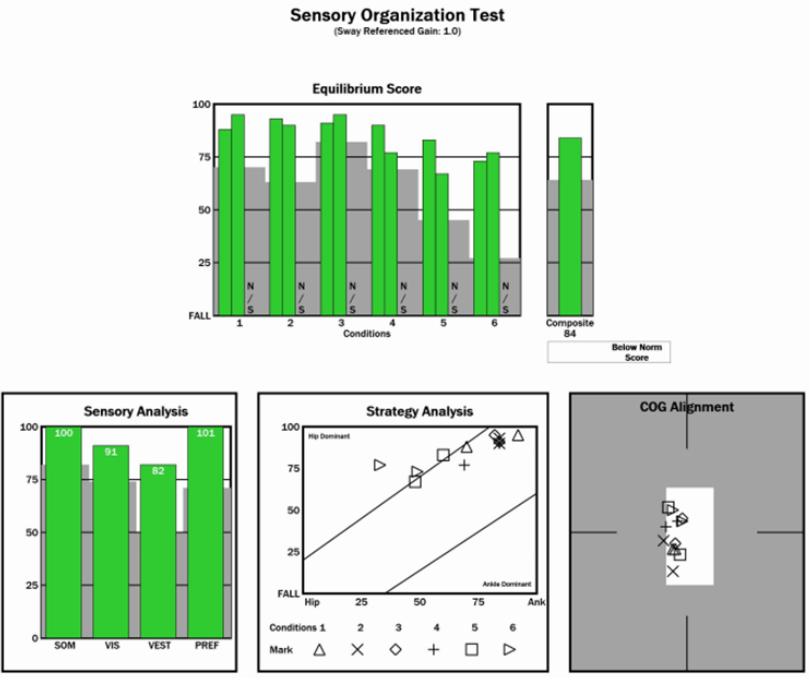

Figure 2B Post-treatment SOT findings on CDP are well within normal limits when compared to age and height matched peers.

The vestibulo-ocular reflex (VOR) was assessed via videonystagmography (VNG), kinetic rotary chair evaluation, and bithermal caloric irrigations. VNG testing was negative for any spontaneous, positionally provoked, gaze evoked, or post-head shaking nystagmus. Both step velocity stimulation and bithermal caloric irrigation yielded bilaterally absent VOR responses, indicative of bilateral vestibular hypofunction. Figure 3 discusses the results during the step velocity rotational assessment. Figure 4 discusses the results during bithermal caloric irrigation evaluation.

\section{Physiotherapy examination}

The patient was seen one week following his neurodiagnostic evaluation in our physiotherapy department. His examination consisted of a review of systems which noted nothing of significance. He underwent functional testing consisting of the Timed Up and Go (TUG) test, 30 Second Sit to Stand test, and manual muscle testing. The Timed Up and Go test is a well-documented functional test looking at assessing falls risk, gait, balance, and mobility. The patient underwent three repetitions of the test with an average score of 10.28 seconds. A TUG score of greater than 13.5 seconds indicates a falls risk for patients with bilateral vestibular hypofunction. 2 His 30 Second Sit to Stand test score was 9 repetitions. The mean average for community-dwelling individuals in his age range (70-79) was 14 repetitions. ${ }^{15}$ Manual muscle testing of his lower extremities were performed with measurement standards by Kendall and McCreary grading. ${ }^{16}$ Overall, his weakness was most noted into hip extension and abduction bilaterally, graded at a $4 / 5$ with his left hip extension at a $3+/ 5$. While these measures do not correlate significantly with falls risk, they were important at assessing the patient's subjective reporting of fatigue, which is highly correlated with falls risk. ${ }^{17-19}$

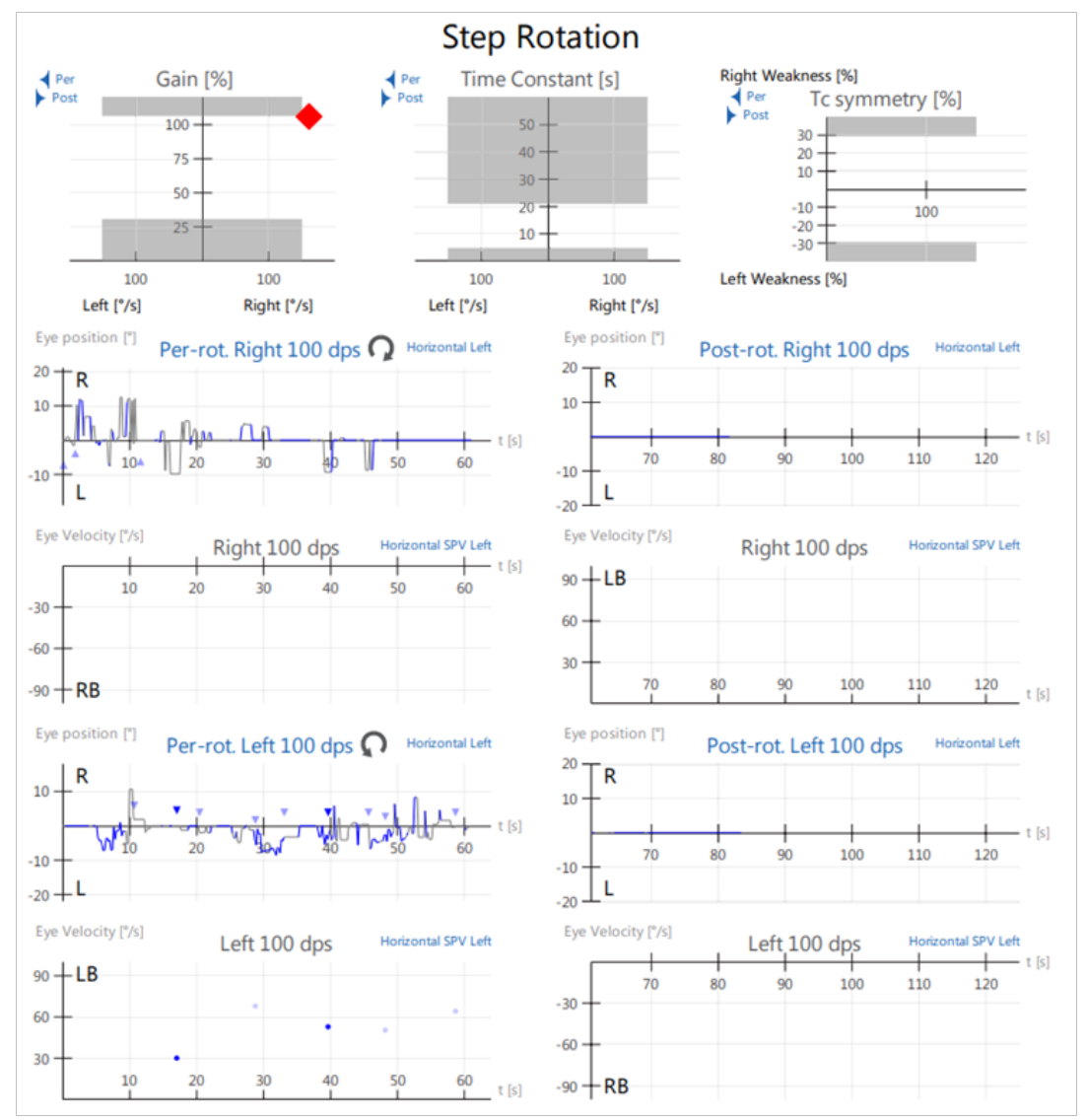

Figure 3 Step velocity rotational testing (completed at 100 degrees/second) at initial evaluation shows no vestibulo-ocular reflex (VOR) gain to bidirectional rotation. 


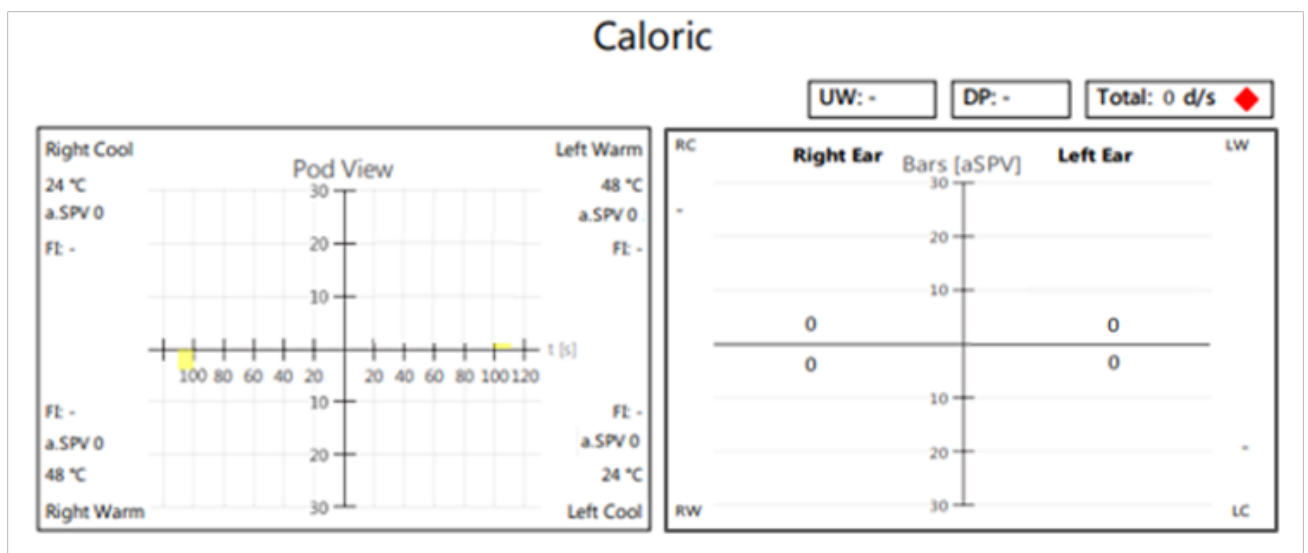

Figure 4 Absent responses to bithermal caloric irrigations, bilaterally, indicating complete bilateral vestibular loss with resulting significant balance dysfunction.

\section{Intervention}

All therapy sessions were completed in American Sign Language through a clinically proficient provider. Interventions were geared towards functional deficits noted in the objective examination. Based on the clinical findings, a mixture of balance retraining therapy and strengthening were most appropriate, consistent with the principle of substitution. After assessing the patient's irritability regarding fatigue and imbalance, the physiotherapist was able to determine an appropriate plan of care and exercise regimen individually tailored to the patient. These exercises ranged from lower extremity strengthening to balance retraining and core stabilization. For the first week, the exercises were specific to lower extremity and lumbar spine, including strengthening and stretching. Subsequent visits following, he was progressed into static standing exercises for hip strengthening and for focus on balance. Our progression choices were made based on constant reassessment in the clinic for his presentations and response to ongoing assessments for his balance reactions. Based on his Sensory Organization Test and Gans SOP, it was determined for specificity in neutral base of support with eyes open were our starting points with a focus on reducing the base of support through narrow stance, semi-tandem, and sharpened tandem stances. Further progression in this yield greater benefit once tolerable into increased surface compliance and eventually eyes closed. By the end of his plan of care, the patient was able to stand on the AIB Balance Performance Foam in tandem with eyes closed for more than 20 seconds. Each of these exercises were assessed for independent performance and follow-through at each visit. Table 2 shows the matched impairmentinterventions that were chosen for this patient.

Table 2 Summary of Paired Impairments and Interventions

\begin{tabular}{|c|c|c|c|c|c|}
\hline Impairments & $\begin{array}{l}\text { Intervention } \\
\text { (week I;2 visits) }\end{array}$ & $\begin{array}{l}\text { Intervention } \\
\text { (week 2; } 2 \text { visits) }\end{array}$ & $\begin{array}{l}\text { Intervention } \\
\text { (week 3; } 2 \text { visits) }\end{array}$ & $\begin{array}{l}\text { Intervention } \\
\text { (week 4; I visit) }\end{array}$ & $\begin{array}{l}\text { Intervention (week } \\
\text { 5; Discharge) }\end{array}$ \\
\hline LE Weakness & $\begin{array}{l}\text { Sit to Stand using } \\
\text { armrest; } 2 \times 10 \\
\text { Bridging; }|x| 5\end{array}$ & $\begin{array}{l}\text { Standing Hip 4-way } \\
\text { x I5 Single Limb } \\
\text { Bridges, Ix I5 }\end{array}$ & $\begin{array}{l}\text { Continued previous } \\
\text { weeks; did not add }\end{array}$ & $\begin{array}{l}\text { Increased repetitions } \\
(B I D) \text { Wall push-up in } \\
\text { SLS } \times 10 \text { ea' leg }\end{array}$ & $\begin{array}{l}\text { Reviewed HEP for } \\
\text { previous weeks and } \\
\text { insured independence. }\end{array}$ \\
\hline Static Balance & $\begin{array}{l}\text { Narrow BOS; 10s } \\
\times 5 \text { Wide BOS; } 20 \text { s } \\
\times 5\end{array}$ & $\begin{array}{l}\text { Wide BOS on } \\
\text { Performance } \\
\text { Foam (PF), } x \text { 10s } x \\
\text { IONarrow BOS on } \\
\text { PF } \times 5 s \times 10\end{array}$ & $\begin{array}{l}\text { Tandem }(\mathrm{B}) \times 10 \mathrm{~s} \times \\
3 \text { Tandem (posterior } \\
\text { leg) on dynadisc } \times \\
10 \mathrm{~s} \times 10\end{array}$ & $\begin{array}{l}\text { Tandem }(\mathrm{B}) \text { on } \mathrm{PF} \times \\
\mathrm{IOs} \times 3 \text { progressing } \\
\text { to } 20 \mathrm{~s}\end{array}$ & $\begin{array}{l}\text { Reviewed HEP for } \\
\text { previous weeks and } \\
\text { insured independence. }\end{array}$ \\
\hline $\begin{array}{l}\text { Dynamic } \\
\text { Balance }\end{array}$ & $\begin{array}{l}\text { Marching in place; } \\
20 \text { s Marching in } \\
\text { place; } 5 \text { s without } \\
\text { holding } \times 4\end{array}$ & $\begin{array}{l}\text { Walking on } \\
\text { compliant mat ( } 10 \mathrm{ft}) \\
\times 10 \text { Ambulating with } \\
\text { turns }(15 \mathrm{ft}) \times 10\end{array}$ & $\begin{array}{l}\text { Walking on } \\
\text { compliant mat with } \\
\text { head rotations } \\
\text { (horizontal) (I cps) } \\
(10 \mathrm{ft}) \times 10\end{array}$ & $\begin{array}{l}\text { Continued from the } \\
\text { previous week. }\end{array}$ & $\begin{array}{l}\text { Reviewed HEP for } \\
\text { previous weeks and } \\
\text { insured independence. }\end{array}$ \\
\hline $\begin{array}{l}\text { Functional } \\
\text { Activities }\end{array}$ & $\begin{array}{l}\text { Combined standing } \\
\text { and reaching } \\
\text { overhead }\end{array}$ & $\begin{array}{l}\text { Forward Bending / } \\
\text { Lifting from ground; } \\
4.5 \mathrm{~kg}, 2 \times 15\end{array}$ & $\begin{array}{l}\text { Bending/Lifting/ } \\
\text { Twisting with } 4.5 \mathrm{~kg} \\
\text { weight }\end{array}$ & $\begin{array}{l}\text { Curb / Stair } \\
\text { management with } \\
\text { visual complexities. }\end{array}$ & $\begin{array}{l}\text { Reviewed HEP for } \\
\text { previous weeks and } \\
\text { insured independence. }\end{array}$ \\
\hline
\end{tabular}

Abbreviations: LE, Lower Extremity; BOS, Base of Support; PF, Performance Foam; (B), bilateral; cPs, cycles per second; BID, twice per day; HEP, Home Exercise Program 


\section{Discussion}

Research demonstrates that while compensation may not be reflected by return of VOR gain in objective testing (i.e. velocitystep rotational testing and caloric testing), functional impairments can improve and/or completely resolve, and objective equilibrium studies can return to normal following rehabilitation; $;{ }^{20}$ however it is important to note that comorbidities can impede the rehabilitation process. ${ }^{21}$ This patient received a structured, multidisciplinary evaluation and treatment plan for his complaints of imbalance and subjective deconditioning.

While we may not know the genesis of the bilateral dysfunction, through accurate diagnosis, proper triage, and a collaborative interdisciplinary approach we were able to focus on the patient's functional deficits and create a targeted rehabilitation program. The success of this program allowed for the patient to return to his daily activities four weeks post his initial evaluation. His fear avoidance completely subsided and he was even able to schedule a deep-sea fishing excursion just a few months after discharge.

\section{Conclusion}

This bilateral vestibulopathy case described the diagnosis and management of a patient with significant balance impairment and resulting deconditioning. Factors that assisted in the clinical decision making model were (1) a thorough examination including subjective questionnaire, case history, and functional concerns, (2) neurodiagnostic testing for inner ear pathologies, (3) communication with the vestibular Audiologist and Physiotherapist, and (4) incorporation of evidence-based treatment for diagnosis of bilateral vestibular impairment, functional impairment, and fall risk management to allow him to return safely to his hobbies and everyday activities.

\section{Acknowledgements}

None.

\section{Conflicts of interest}

No potential conflict of interest relevant to this article was reported.

\section{References}

1. Hall CD, Herdman SJ, Whitney SL, et al. Vestibular rehabilitation for peripheral vestibular hypofunction: an evidence-based clinical practice guideline. J Neurol Phys Ther. 2016;40(2):124-155.

2. Crespi V. Dizziness and vertigo: an epidemiological survey and patient management in the emergency room. Neurol Sci. 2004;25 Suppl 1:S24 S25.

3. Jahn K, Kreuzpointner A, Pfefferkorn T, et al. Telling friend from foe in emergency vertigo and dizziness: does season and daytime of presentation help in the differential diagnosis?. J Neurol. 2020:11.

4. Royl G, Ploner CJ, Leithner C. Dizziness in the emergency room: diagnoses and misdiagnoses. Eur Neurol. 2011;66(5):256-263.
5. Important facts about falls | home and recreational safety $\mid \mathrm{CDC}$ injury center. 2016.

6. Costs of falls among older adults | home and recreational safety | CDC injury center. 2016.

7. Gans RE. Vestibular rehabilitation: protocols and programs. Singular Publishing Group; 1996.

8. Gans R. Vestibular rehabilitation. Textbook of vertigo: Diagnosis and management. 2013:245.

9. Porciuncula F, Johnson CC, Glickman LB. The effect of vestibular rehabilitation on adults with bilateral vestibular hypofunction: A systematic review. Journal of Vestibular Research. 2012;22(5,6):283298

10. Gans RE, Rauterkus G. Pharmacology and ototoxicity: vestibular toxicity: causes, evaluation protocols, intervention, and management. IN: seminars in hearing. Thieme Medical Publishers. 2019(40):144.

11. Brown KE, Whitney SL, Wrisley DM, et al. Physical therapy outcomes for persons with bilateral vestibular loss. Laryngoscope. 2001;111(10):1812-1817.

12. Whitney SL, Wrisley DM, Brown KE, et al. Is perception of handicap related to functional performance in persons with vestibular dysfunction?. Otol Neurotol. 2004;25(2):139-143.

13. Jacobson GP, Newman CW, Hunter L, et al. Balance function test correlates of the dizziness handicap inventory. J Am Acad Audiol. 1991;2(4):253-260.

14. Krebs DE, Gill-Body KM, Riley PO, et al. Double-blind, placebocontrolled trial of rehabilitation for bilateral vestibular hypofunction: preliminary report. Otolaryngol Head Neck Surg. 1993;109(4):735-741.

15. Rikli RE, Jones CJ. Development and validation of criterionreferenced clinically relevant fitness standards for maintaining physical independence in later years. Gerontologist. 2013;53(2):255-267.

16. Kendall FP, McCreary EK, Provance PG, et al. Others. muscles, testing and function: with posture and pain. Williams \& Wilkins Baltimore, MD; 1993:103.

17. Bobowik P, Wiszomirska I. Diagnostic dependence of muscle strength measurements and the risk of falls in the elderly. International Journal of Rehabilitation Research. 2020.

18. Helbostad JL, Sturnieks DL, Menant J, et al. Consequences of lower extremity and trunk muscle fatigue on balance and functional tasks in older people: a systematic literature review. BMC Geriatr. 2010;10:56.

19. Parijat $P$, Lockhart TE. Effects of lower extremity muscle fatigue on the outcomes of slip-induced falls. Ergonomics. 2008;51(12):1873-1884.

20. Gillespie MB, Minor LB. Prognosis in bilateral vestibular hypofunction. Laryngoscope. 1999;109(1):35-41.

21. Herdman SJ, Hall CD, Maloney B, et al. Variables associated with outcome in patients with bilateral vestibular hypofunction: Preliminary study. J Vestib Res. 2015;25(3-4):185-194. 